\title{
Practical approaches to verification and validation of engineering software when solving problems of nonlinear and chaotic dynamics
}

\author{
Anna Voronova ${ }^{1}$, Elena Zhilenkova ${ }^{2}$, Anton Zhilenkov ${ }^{1,}{ }^{*}$, and Vladislav Borisenko ${ }^{2}$ \\ ${ }^{1}$ Saint Petersburg State Marine Technical University, 190121, St. Petersburg, Russia \\ ${ }^{2}$ Peter the Great St. Petersburg Polytechnic University, 195251, St. Petersburg, Russia
}

\begin{abstract}
The article discusses the problem of verification and validation of engineering software when solving problems of nonlinear dynamics. The problems of validation and verification are shown on the example of dynamic chaos systems. The results of testing systems are presented. It is shown that in general when solving problems of nonlinear dynamics the characteristics of the developed engineering software are of critical importance. It is also indicated that neglecting this fact leads to irreversible negative consequences, ultimately resulting in the decay of the dynamics of a nonlinear system as well as in the decay of its orbits. The influence of the hardware and a number of aspects of the system and software implementation of the verification and validation systems under study is also shown. The article demonstrates modern approaches to the development of the studied software systems. It is shown that a high-quality software product suggests division into subsystems and stages.
\end{abstract}

\section{Introduction}

The infrastructure of the system under consideration is designed to isolate critical system components from servers to distribute the load and eliminate errors in software and hardware.

The main objective of study is to prove the reliability of the output of verification and validation programs for systems with nonlinear dynamics. The so-called dynamic chaos systems were selected for testing. Although these systems are continuous in their behavior, they demonstrate little predictable dynamics of the output. It is known that the behavior of such systems is heavily dependent on the initial conditions. On the other hand, according to the well-known shadowing theorem, small deviations from the orbit do not lead to a significant change in the system's behavior. These facts relate to continuous dynamic systems with nonlinear dynamics. There have been few detailed studies on the behavior of discrete or digital systems under these conditions. It is clear that the features of the implementation of the software of the validation and verification systems, as well as a number of aspects of the hardware, can significantly affect not only the deviation of the initial conditions of a nonlinear dynamic system, but also the deviation of its orbits from the orbits observed in the case of continuous systems.

\footnotetext{
* Corresponding author: zhilenkovanton@gmail.com
} 
Thus, based on a single analytical description of nonlinear dynamics systems demonstrating complex and difficult to predict behavior, one can expect that the quality and reliability of modeling their long-term dynamics, statistics, or the density of the output probability distribution will critically depend on the numerical methods used, the features of computer arithmetic, and the accuracy of calculations and the features of the hardware on which the platform is deployed.

\section{Problems of implementation of digital complexes for testing systems with nonlinear dynamics}

As the developer starts to implement the described scheme of validation and verification system of nonlinear dynamics systems, he faces the problem of the accuracy of data presented in digital systems, and the problems of quantization and rounding data. It directly affects the accuracy of modeling systems with nonlinear dynamics, which is demonstrated in the following test models.

The first example that clearly demonstrates the problems of modeling and verification of a nonlinear dynamic system based on a digital platform is a piecewise linear function of the "tent" type, represented as the following system $[1,2,4,6,8]$ :

$$
f(x, \lambda)=\left\{\begin{array}{cc}
\frac{x}{\lambda}, & 0 \leq x<\lambda \\
\frac{x-\lambda}{0.5-\lambda}, & \lambda \leq x \leq 0.5 \\
f(1-x, \lambda), & 0.5 \leq x<1 .
\end{array}\right.
$$

Let us investigate this system with the parameter $\lambda=0.321$ and the initial value $x_{0}=$ 0.00485 .

Below are the graphs $y(x)$ (Fig. 1-3) with the number of iterations $i=2000$ for the case when no additional rounding was applied. The following graphs are also presented:

- with rounding through the floor function,

- with rounding through the ceil function,

- with rounding through the round function,

where rounding is performed to the fourth decimal place. 


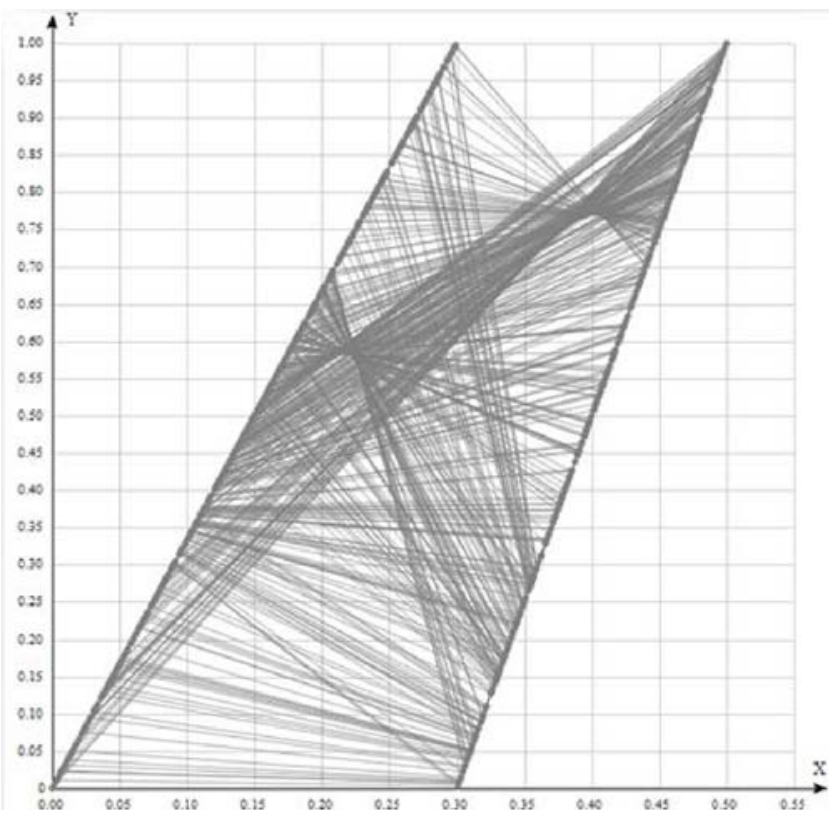

Fig. 1. Graph of the function $\mathrm{Y}(\mathrm{x})$ of the "tent" type without rounding.

The construction of a graph of the output of this and subsequent systems with nonlinear dynamics has the main purpose of demonstrating the decay of the dynamics of their output and statistical indicators reflecting the unevenness of the probability distribution density [3, 5].

It is expected that the features of the hardware implementation lead to the orbital decay of the simulated nonlinear systems, which is confirmed by numerical simulation. The results of such a numerical experiment are shown in Figure 2.

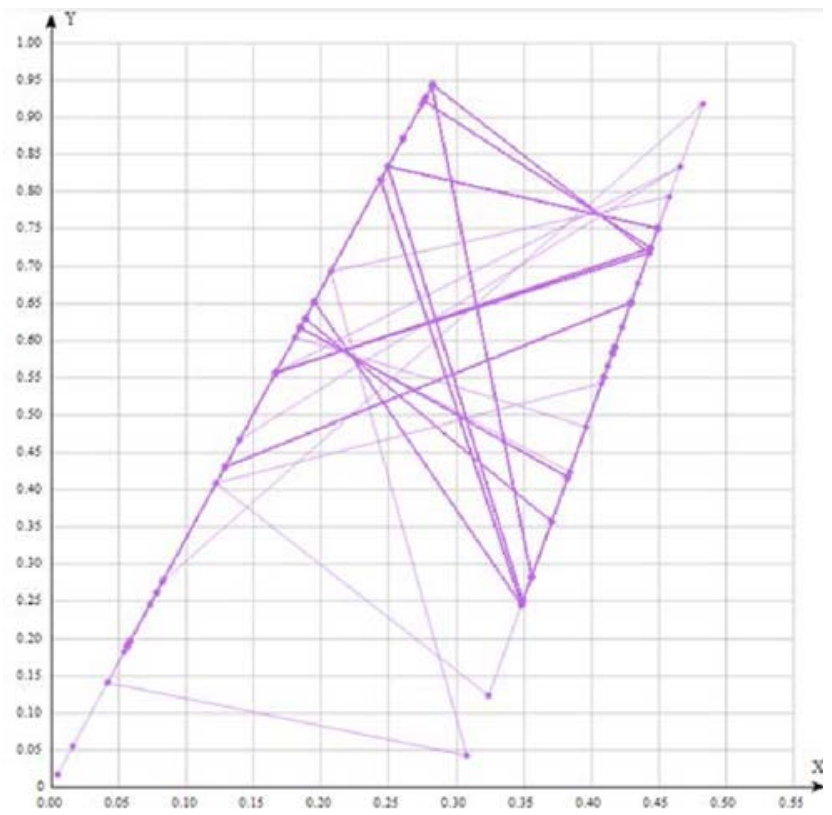

Fig. 2. Graph of the function $\mathrm{Y}(\mathrm{x})$ of the "tent" type without rounding. 
As can be seen in Figure 1, the range of values of the function is evenly distributed and attractors of the system are observed. In Figure 2 it is clearly seen that the same function has a different distribution of values in the same area. Since in the second experiment (Fig. 2) the number of iterations was the same as in the first one (Fig. 1), it is obvious that in Fig. 2 there is a multiple repeating and looping orbits and, as will be shown below, in a number of cases, degenerating to a fixed point.

Similar experiments carried out for the rounding options described above when calculating the values of the same function describing a nonlinear system are shown in Figures 3-5.

When comparing the graphs in Fig. 1-4, it is clearly seen that when rounding, the number of different orbits on the graph is significantly reduced and the repetition of certain orbits increases $[4,6,8]$. This eventually leads to the predictability of the system due to an increase in the periodicity and, in fact, an increase in the heterogeneity of the density of the output probability distribution. When using the specified "tent" function as a random number generator, such decay leads to a critical decrease in the cryptographic strength of the system, which can be provided only in the case of minimal predictability of its output.

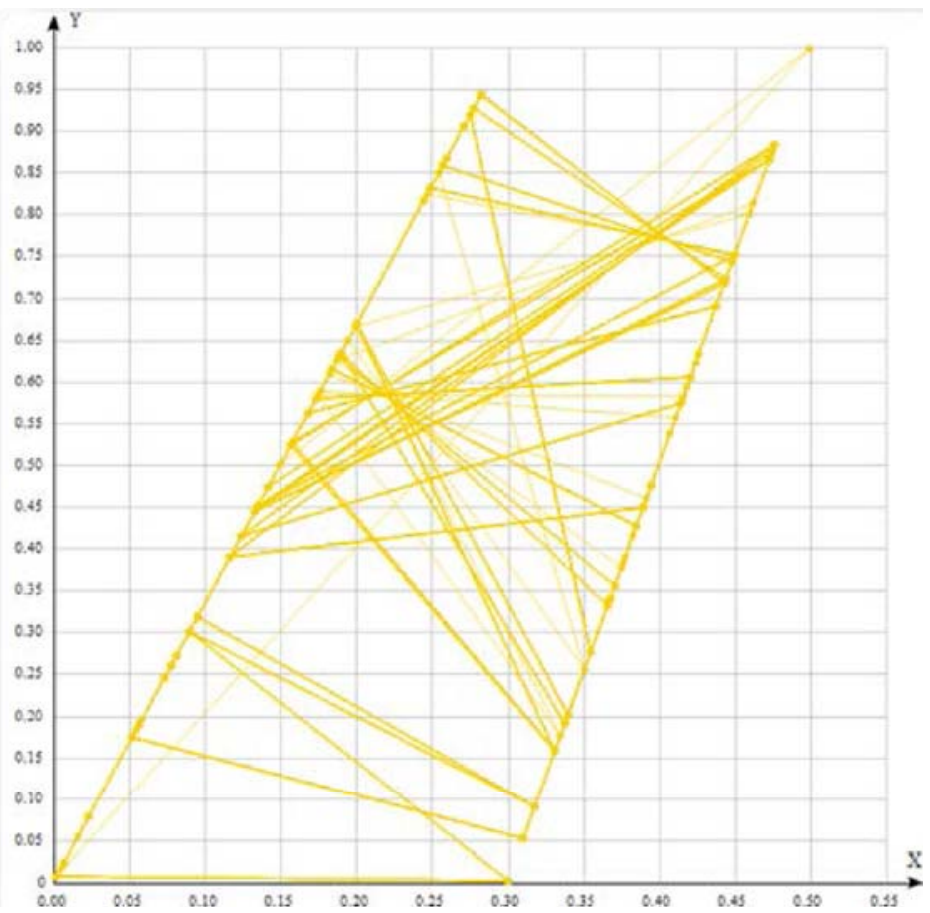

Fig. 3. Graph of the function $\mathrm{Y}(\mathrm{x})$ of the "tent" type with rounding through the ceil function. 


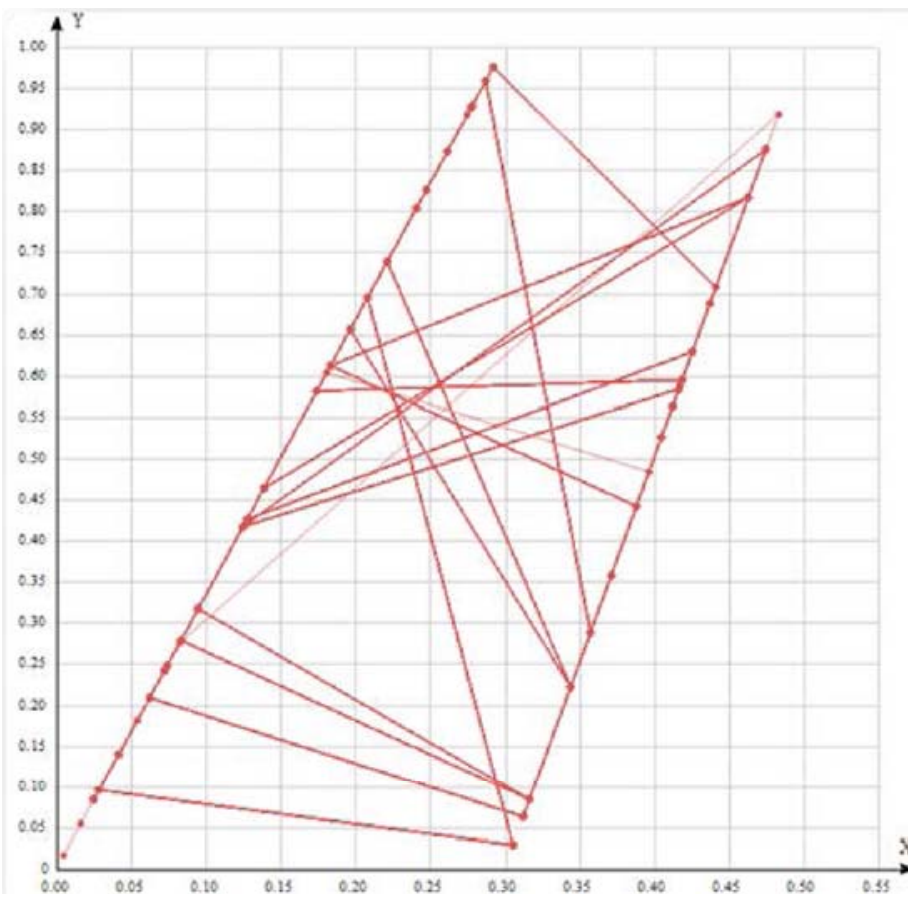

Fig. 4. Graph of the function $\mathrm{Y}(\mathrm{x})$ of the "tent" type with rounding through the round function.

As a second example, let's examine the function represented by the following formula:

$$
f(x, \lambda)=\left\{\begin{array}{l}
\frac{x}{\lambda}, 0 \leq x \leq \lambda \\
\frac{1-x}{1-\lambda}, \lambda<x \leq 1 .
\end{array},\right.
$$

We investigate the behavior of this system with the parameter $\lambda=0.625$ and the initial value $x_{0}=0.01975$.

The graphs of $y(x)$ for the number of iterations $i=2000$ without rounding, using the rounding floor, ceil and round functions to 4 decimal places after the point are provided below. 


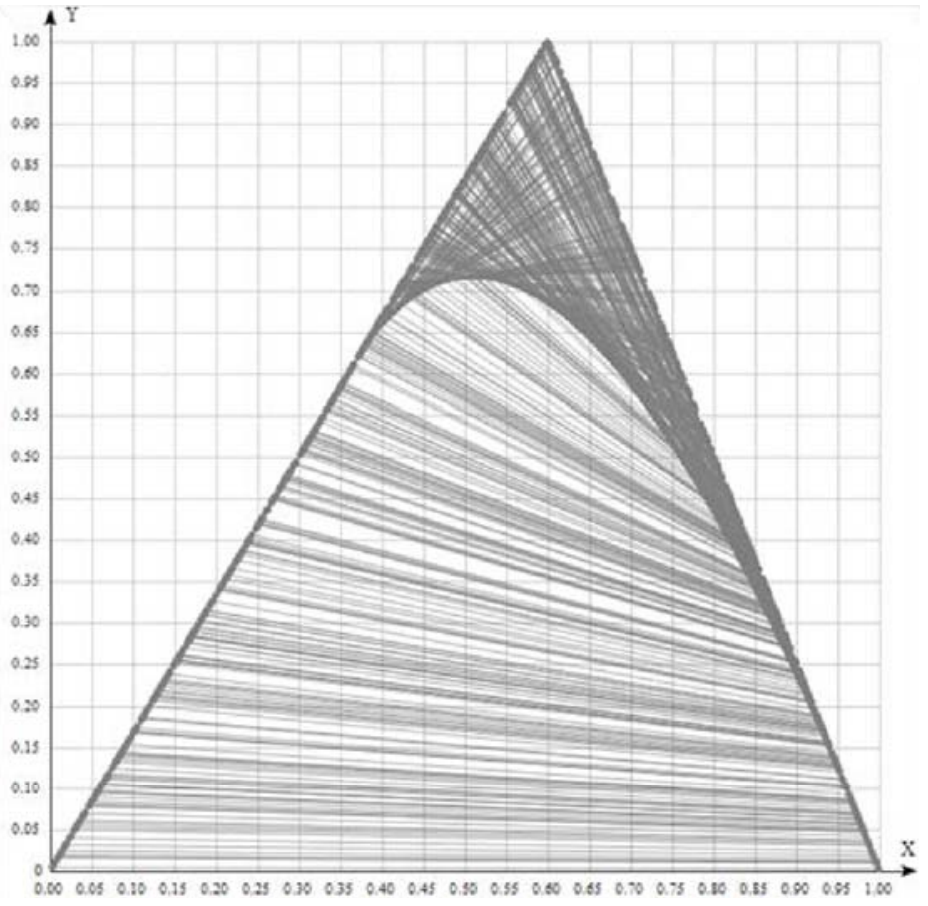

Fig. 5. Graph of $y(x)$ function No.2 without rounding.

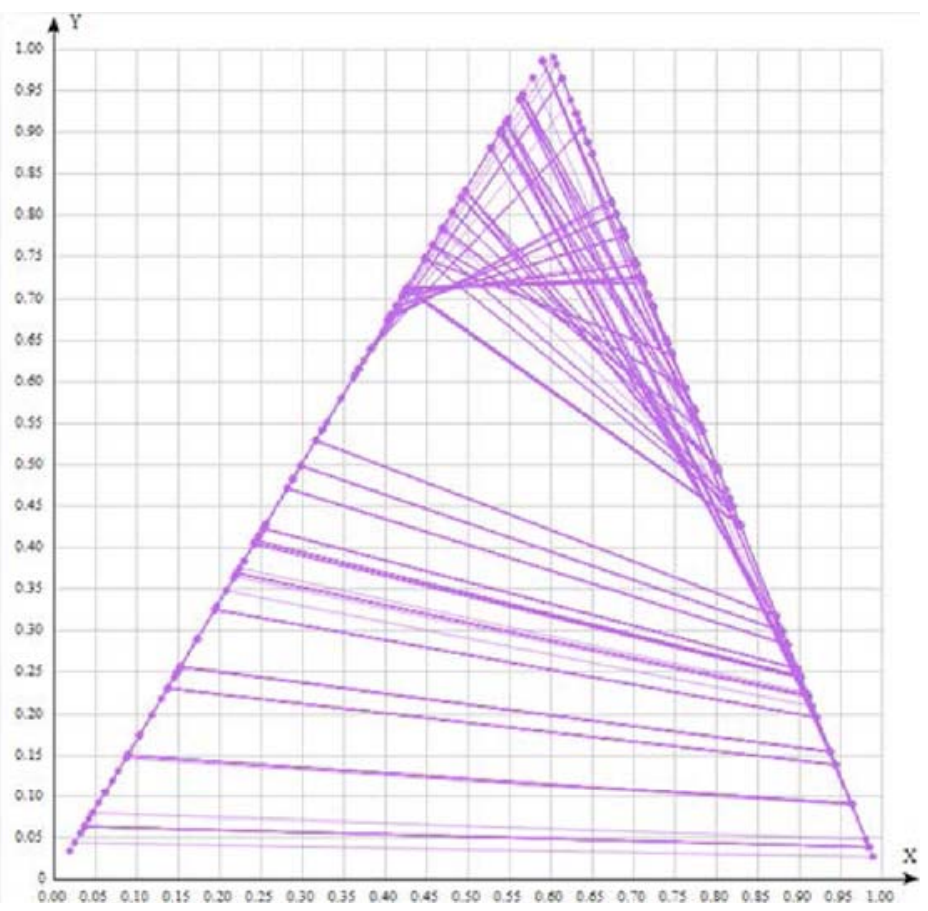

Fig. 6. Graph of $y(x)$ function No.2 with floor rounding. 


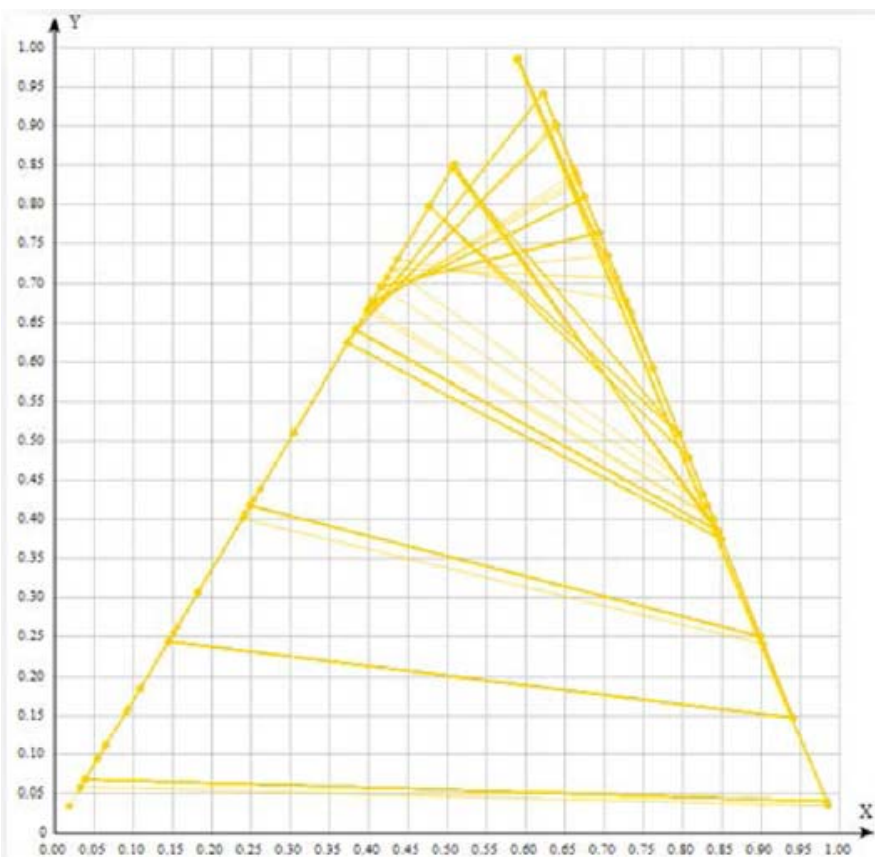

Fig. 7. Graph of $\mathrm{y}(\mathrm{x})$ function No.2 with ceil rounding.

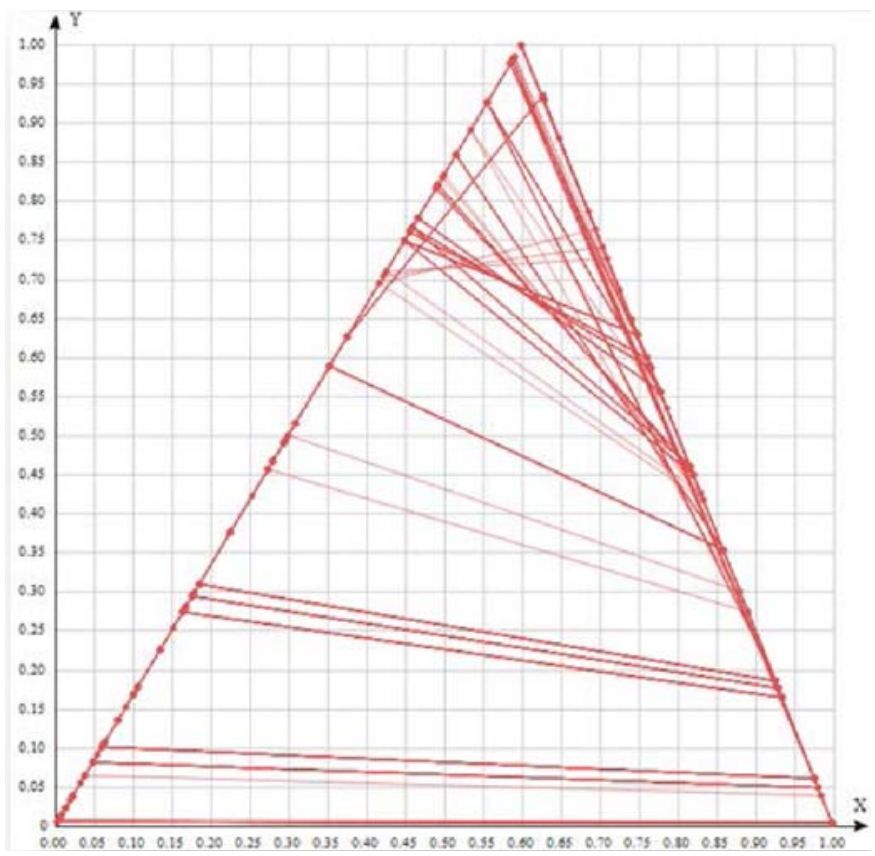

Fig. 8. Graph of $y(x)$ function No.2 with rounding.

When analyzing the graphs in Fig. 6-9, changes are clearly visible both in the area of the vertex of the figure and throughout its entire area $[9,10]$. Depending on the use of one or another rounding, the number of non-repeating lines is either reduced or increased.

As can be seen, in each of the cases considered above, there is a critical decay of the dynamics of the system output. It is obvious that such a deviation of the behavior of the 
modeled or verified system from the real one is unacceptable for testing and verification systems. That is also true for modeling systems. As a conclusion, it can also be noted that the implementation of data coding or encryption systems based on systems of nonlinear dynamics (or systems of dynamic chaos) depends mostly on the hardware implementation and the accuracy of arithmetic in it. Anyway, digital systems simulate nonlinear dynamics with significant decay. In encryption systems, for example, this leads to a critical decrease in cryptographic strength due to the appearance of frequently repeated combinations of coding sequences.

\section{The density of the output probability distribution of the investigated nonlinear dynamic systems}

Having considered examples demonstrating the dynamics decay of the output of the systems under study, it is necessary to investigate the statistical indicators of these systems under the same conditions. Let us consider the statistics of the distribution of units over bits in the IEEE-754 format for representing a floating point number in a double-precision binary number system. This experience will clearly show how this or that rounding method affects the density of the output probability distribution of each of the systems under consideration.

The bit distribution statistics when using the first function (output dynamics in Fig. 1-4) described above looks like Figure 9.

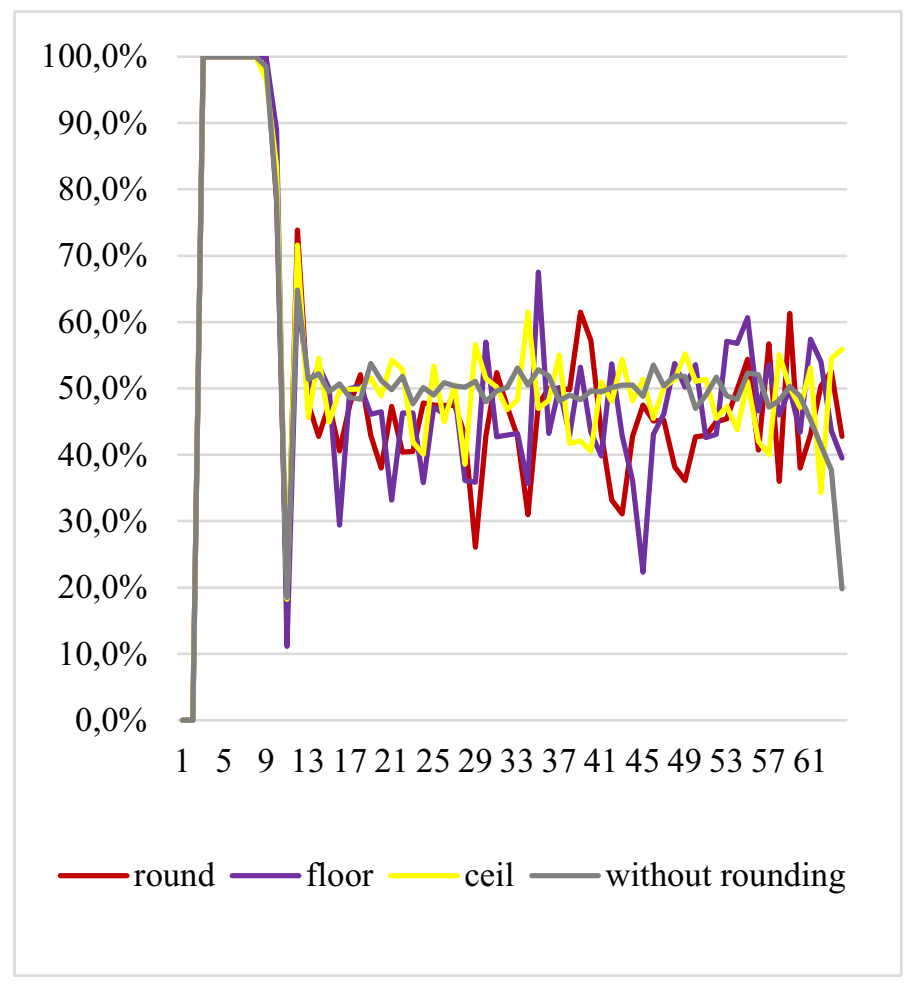

Fig. 9. Bit distribution statistics using function (1). 


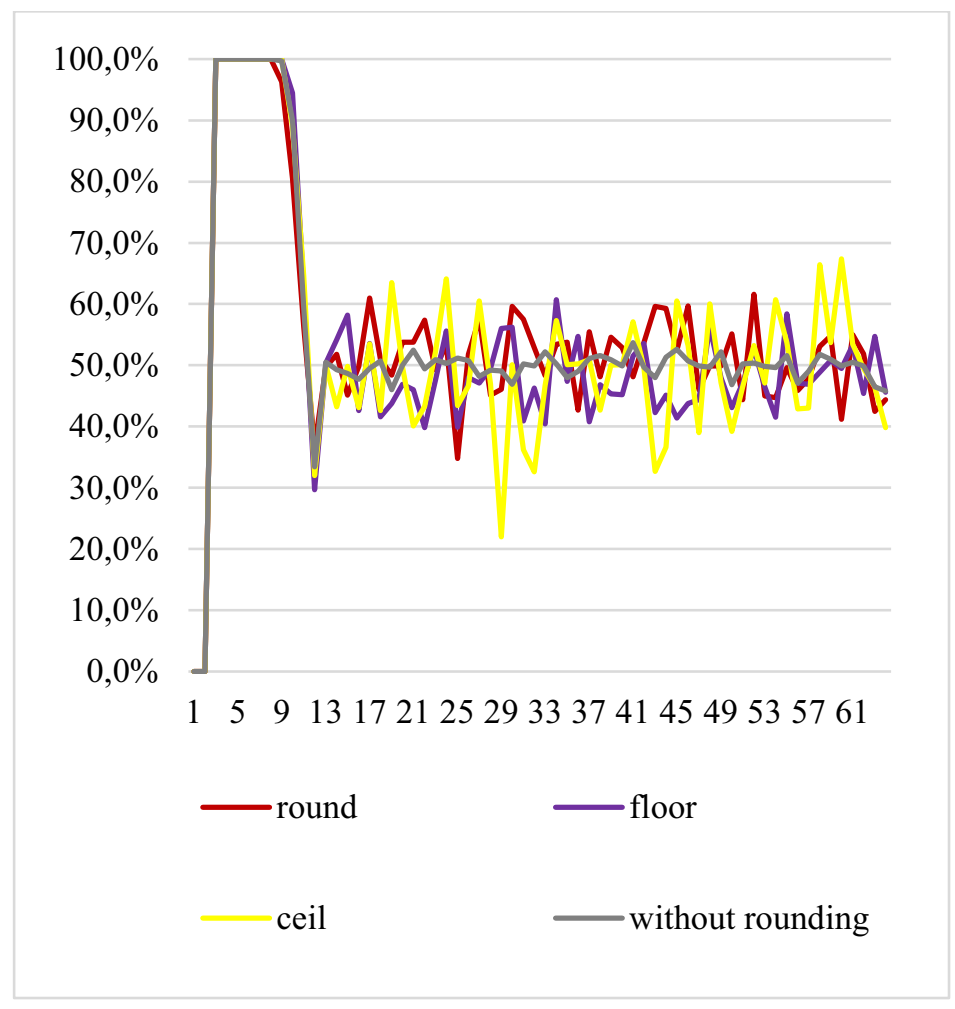

Fig. 10. Bit distribution statistics using function (2).

As can be seen from the graphs in Figs. 11-12, with a positive initial value $\mathrm{x} 0$, the sign of the variables remains the same, that is, positive. However, in this case the distribution of the bits of the mantissa is of particular interest.

\section{Conclusion}

Digital validation and verification systems are in demand for nonlinear dynamic systems during their design and testing. The use of testing based on a mathematical or computer model, on a digital twin, etc., can significantly reduce the cost of designing these systems. This applies to satellite systems, objects created in the aviation and shipbuilding industry, data encryption and encoding systems and many others.

As has been shown in the article, the main problem in the implementation of such digital platforms for verification is the deterioration of the behavior of a nonlinear system when simulated using such platforms. As shown in the graphs above, the nonlinear dynamics system undergoes significant decay of the output dynamics, as well as the decay of statistical indicators of the systems output for the case of deterministic chaos.

The decay of the dynamics is expressed in the looping of the orbits. Thus, they leave the orbits of the systems observed in the absence of restrictions on the accuracy of the hardware simulation system. That is, for the case of continuous systems without quantization.

It is certain that in the case of using floating point systems, the behavior of these systems will be different. However, the results presented in the article show that a number of nonlinear dynamic systems will experience significant decay at the output even in the case of floating point and double precision data representation. 
Analytical assessment and recommendations for the implementation of digital platforms for validation and verification of nonlinear dynamic systems should be carried out and given as a result of separate studies and tests. This article provides the main reasons and consequences of using digital systems for these tasks.

The authors believe that global trends will just make such studies more relevant.

\section{References}

1. D. Kim, H. Peng, S. Bai, J. Maguire, IEEE Transactions on Control System Technology 15(3), 474 (2007)

2. R. Petrov, P. Cvetkov, A. Maksimov, O. Klyavin, Int. J. Mech. 14, 119 (2020)

3. S. Bai, D. Brennan, D. Dusenberry, X. Tao, Z. Zhang, SAE International Paper 201001-0368 (2010)

4. A.A. Zhilenkov, D. Denk, IEEE Conference of Russian Young Researchers in Electrical and Electronic Engineering (EIConRus) $1104 \quad$ (2017) doi: 10.1109/EIConRus.2017.7910748

5. A. Al-Dulaimi, S. Zabihi, A. Asif, A. Mohammadi, Comput. Ind. 108, 186-96 (2019)

6. A.V. Ivanov, A.A. Zhilenkov, IEEE Conference of Russian Young Researchers in Electrical and Electronic Engineering (EIConRus) 890 (2018) doi: 10.1109/EIConRus.2018.8317231

7. A. Bhardwaj, W. Di, J. Wei, Deep Learning Essentials: Your hands-on guide to the fundamentals of deep learning and neural network modeling (Packt Publishing Limited, Birmingham, 2018)

8. S. Sokolov, A. Zhilenkov, A. Nyrkov, S.Chernyi, Advances in Intelligent Systems and Computing 556 (2017) https://doi.org/10.1007/978-981-10-3874-7_39

9. J.T. Barron, Proc. of the IEEE Conf. on Computer Vision and Pattern Recognition (CVPR) (2019)

10. A.D. Karpov, A.A. Zhilenkov, D. Lisitsa, IEEE Conference of Russian Young Researchers in Electrical and Electronic Engineering (EIConRus) 887 (2017) doi: 10.1109/EIConRus.2017.7910697 